\title{
Efecto del nivel proteico de la dieta sobre el desarrollo de juveniles de Macrobrachium tenellum (Smith, 1871)
}

\section{Effect of dietary protein level on the development of juveniles of Macrobrachiun tenellum (Smith, 1871)}

\author{
Luis Espinosa-Chaurand, ${ }^{1}$ M.Sc, Carlos Flores-Zepeda, ${ }^{2}$ Lic, Héctor Nolasco-Soria, ${ }^{3}$ Ph.D, \\ Olimpia Carrillo-Farnes, ${ }^{4}$ Ph.D, Fernando Vega-Villasante, ${ }^{1 *}$ Ph.D.
}

\begin{abstract}
${ }^{1}$ Universidad de Guadalajara. Centro de Investigaciones Costeras. Laboratorio de Acuicultura Experimental. Av. Universidad No. 203, Del. Ixtapa, C.P. 48280, Puerto Vallarta, Jalisco, México. 2Universidad de Guadalajara. Centro Universitario de la Costa. Licenciatura en Biología. Puerto Vallarta, Jalisco, México. ${ }^{3}$ Centro de Investigaciones Biológicas del Noroeste S.C. La Paz, Baja California Sur, México. ${ }^{4}$ Universidad de La Habana. Facultad de Biología. La Habana, Cuba. Correspondencia: fernandovega.villasante@gmail.com
\end{abstract}

Recibido: Diciembre de 2011; Aceptado: Agosto de 2012.

\section{RESUMEN}

Objetivo. Evaluar el efecto de cinco niveles de proteína cruda (PC) en alimentos balanceados sobre el crecimiento, sobrevivencia y tasa de conversión alimenticia (FCA) en juveniles de Macrobrachium tenellum. Materiales y métodos. Se alimentó por 60 días a juveniles de $M$. tenellum $(0.31 \pm 0.01 \mathrm{~g}$ y $32.62 \pm 1.10 \mathrm{~mm}$ ) con niveles de $20,25,30,35$ y $40 \%$ de PC en el alimento. Los organismos fueron distribuidos al azar en 15 tinas experimentales de $64 \mathrm{~L}$ (15 org./tina) bajo condiciones controladas $\left(5.95 \pm 0.41 \mathrm{ppm}\right.$ de oxígeno, $29.89 \pm 0.72^{\circ} \mathrm{C}$, y $\left.\mathrm{pH} 8.44 \pm 0.15\right)$ y alimentados con el $10 \%$ de su peso vivo. Resultados. El porcentaje de sobrevivencia fue del $98.22 \pm 3.96 \%$ sin diferencias significativas entre los tratamientos $(p>0.05)$. Los organismos alimentados con un $40 \%$ de PC tuvieron un peso significativamente mayor $(p<0.05)$ respecto a los demás tratamientos (cambio de peso de $0.54 \pm 0.02 \mathrm{~g}$; incremento de peso de $173.60 \pm 12.99 \%$; y tasa de crecimiento específico de $1.68 \pm 0.08)$. El FCA fue significativamente mejor $(p<0.05)$ en los organismos alimentados con 35 y $40 \%$ de PC $(2.85 \pm 0.18$ y $2.40 \pm 0.05$, respectivamente) que los demás tratamientos. Conclusiones. Los organismos juveniles de $M$. tenellum alimentados con niveles altos de proteína $(40 \%)$, se desarrollaron más rápido que organismos que recibieron una menor concentración de proteína bajo las condiciones experimentales establecidas en este estudio.

Palabras clave: Alimentos, crecimiento, Macrobrachium, nutrición, proteína de la dieta (Fuente: CAB). 


\begin{abstract}
Objective. To evaluate the effect of five levels of crude protein (CP) in balanced feed on the survival, growth and feed conversion ratio (FCA) in juveniles of Macrobrachium tenellum. Materials and methods. Juveniles of $M$. tenellum $(0.31 \pm 0.01 \mathrm{~g}$ and $32.62 \pm 1.10 \mathrm{~mm})$ were fed for 60 days with $20,25,30,35$ and $40 \%$ of CP in feed. The organisms were randomly distributed in 15 experimental tanks (15 org /tank) under controlled conditions (5.95 $\pm 0.41 \mathrm{ppm}$ of oxygen, $29.89 \pm 0.72{ }^{\circ} \mathrm{C}$, and pH $8.44 \pm 0.15$ ) and fed with $10 \%$ of its live weight. Results. The survival percentage was $98.22 \pm 3.96 \%$ with no statistical difference between treatments ( $p>0.05$ ). The organisms fed with $40 \% \mathrm{CP}$ in their diet had a significantly higher weight $(p<0.05)$ compared to the other treatments (weight change of $0.54 \pm 0.02 \mathrm{~g}$; weight increase $173.60 \pm 12.99 \%$, and specific growth rate of $1.68 \pm 0.08)$. The FCA was significantly better $(p<0.05)$ in organisms fed with 35 and $40 \%$ CP $(2.85 \pm 0.18$ and $2.40 \pm 0.05$, respectively) than other treatments. Conclusions. Juveniles of $M$. tenellum fed with high protein levels $(40 \%)$ developed faster than organisms which received a lower concentration of the protein under the experimental conditions established for this study.
\end{abstract}

Key words: Dietary protein, foods, growth, Macrobrachium, nutrition (Source: $C A B$ ).

\section{INTRODUCCIÓN}

De los camarones de agua dulce nativos en América existen aproximadamente 26 especies en México, América Central y Sudamérica (1), de las cuales se destacan cuatro especies de importancia comercial Macrobrachium carcinus y $M$. acanthurus en el Atlántico y M. tenellum y M. americanum en el Pacífico $(1,2)$. A partir de la segunda mitad de la década de los setentas el langostino $M$. tenellum fue considerado como un buen candidato para ser cultivado, teniendo en cuanta que en condiciones naturales se encuentra en altas densidades, no es agresivo y tolera un amplio y fluctuante intervalo de temperatura, salinidad y concentraciones de oxígeno (1).

En un cultivo exitoso de langostino, el manejo y la alimentación son considerados de gran importancia, puesto que el alimento constituye del 40 al $60 \%$ de los costos de producción (3). El tipo de alimento y la estrategia de alimentación representan factores de vital importancia en el aporte de la energía y nutrientes necesarios para el adecuado desarrollo (4) y crecimiento de organismos acuáticos $(4,5)$. En alimentos prácticos formulados para la acuicultura, la proteína es el componente energético más caro y su calidad representa un aspecto nutricional muy importante (6), ya que esta es utilizada por el organismo con tres fines fundamentales: mantenimiento, recuperación de los tejidos dañados y crecimiento (7).

Pese a los esfuerzos realizados con relación al estudio de la nutrición de $M$. tenellum, aún no se conocen los requerimientos para cada una de sus etapas de cultivo (2). Entre estos estudios se ha informado que en la sustitución proteica de harina de pesado por harina de soya en las dietas de M. tenellum, no han sido afectados los parámetros productivos e índices de crecimiento por dicha sustitución (7), además se ha indicado por algunos investigadores la necesidad de desarrollar una dieta nutricionalmente balanceada con el objetivo de optimizar la producción del $M$. tenellum (1). Por todo lo anterior, es necesario determinar el nivel de proteína adecuado en la dieta para un óptimo crecimiento de juveniles de M. tenellum.

El objetivo del presente trabajo fue evaluar el efecto del nivel de proteína $(20,25,30,35$ y $40 \%$ de PC) en la dieta sobre el crecimiento, sobrevivencia y tasa de conversión en juveniles de esta especie.

\section{MATERIALES Y MÉTODOS}

Tipo de estudio. Se realizó un estudio de tipo experimental entre julio y septiembre de 2011.

Sitio de estudio. El presente estudio se llevó a cabo en el Laboratorio de Acuicultura Experimental del Centro Universitario de la Costa de la Universidad de Guadalajara (CUCOSTA), localizado en La Delegación Ixtapa, municipio de Puerto Vallarta, Jalisco, México (20042 '19' ' $\mathrm{N}$ y $105^{\circ} 13^{\prime} 16^{\prime \prime}$ 'O) a una altitud de 10 metros sobre el nivel medio del mar.

Dietas experimentales empleadas. Se elaboraron cinco dietas experimentales con niveles de proteína cruda (PC) de $20 \%, 25 \%$, $30 \%$, 35\% y $40 \%$ (Tabla 1 ). Para la elaboración de las dietas se utilizó como principal fuente de proteína la harina de pescado. Todos los ingredientes fueron mezclados en un procesador de alimentos (Hobart, Troy, $\mathrm{OH}$ ) durante 10 15 min, hasta producir una masa homogénea. 
Tabla 1. Ingredientes y composición proximal de las dietas formuladas ( $\mathrm{g} / 100 \mathrm{~g}$ de peso seco).

\begin{tabular}{|c|c|c|c|c|c|}
\hline \multirow{2}{*}{$\begin{array}{l}\text { Ingredientes } \\
(\mathrm{g} / 100 \mathrm{~g})\end{array}$} & \multicolumn{5}{|c|}{ Nivel de proteína en los alimentos experimentales } \\
\hline & $20 \%$ & $25 \%$ & $30 \%$ & $35 \%$ & $40 \%$ \\
\hline Harina integral trigo & 38.00 & 38.00 & 38.00 & 38.00 & 35.09 \\
\hline Almidón de maíz & 27.18 & 19.82 & 12.47 & 5.11 & 0.00 \\
\hline Pasta de soya & 10.00 & 10.00 & 10.00 & 10.00 & 10.00 \\
\hline Harina de pescado & 9.11 & 16.82 & 24.58 & 32.31 & 40.67 \\
\hline Aceite de hígado de bacalao & 5.71 & 5.33 & 4.96 & 4.58 & 4.24 \\
\hline Harina de calamar & 5.00 & 5.00 & 5.00 & 5.00 & 5.00 \\
\hline Premezcla mineral crustáceos ${ }^{a}$ & 2.50 & 2.50 & 2.50 & 2.50 & 2.50 \\
\hline Carbonato de calcio & 1.00 & 1.00 & 1.00 & 1.00 & 1.00 \\
\hline Lecitina de soya & 1.00 & 1.00 & 1.00 & 1.00 & 1.00 \\
\hline Premezcla vitamina crustáceos ${ }^{b}$ & 0.30 & 0.30 & 0.30 & 0.30 & 0.30 \\
\hline Vitamina C & 0.10 & 0.10 & 0.10 & 0.10 & 0.10 \\
\hline Cloruro de colina & 0.10 & 0.10 & 0.10 & 0.10 & 0.10 \\
\hline \multicolumn{6}{|c|}{ Composición proximal real ( $\mathrm{g} / 100 \mathrm{~g}$ en peso seco) } \\
\hline Proteína cruda $(\mathrm{N} \times 6.25)$ & $20.79 \pm 0.27$ & $26.41 \pm 0.04$ & $32.01 \pm 0.16$ & $37.63 \pm 0.19$ & $42.55 \pm 0.23$ \\
\hline Lípidos totales & $7.89 \pm 0.12$ & $7.86 \pm 0.13$ & $8.15 \pm 0.07$ & $7.99 \pm 0.04$ & $8.47 \pm 0.10$ \\
\hline Fibra cruda & $0.67 \pm 0.06$ & $0.43 \pm 0.03$ & $0.47 \pm 0.05$ & $0.36 \pm 0.06$ & $0.34 \pm 0.06$ \\
\hline Cenizas & $6.55 \pm 0.04$ & $7.84 \pm 0.05$ & $9.04 \pm 0.02$ & $10.52 \pm 0.02$ & $11.73 \pm 0.01$ \\
\hline Extracto libre nitrógeno ${ }^{c}$ & 64.11 & 57.46 & 50.32 & 43.50 & 36.91 \\
\hline Energía (Kcal g-1) & $4.36 \pm 0.03$ & $4.42 \pm 0.01$ & $4.55 \pm 0.02$ & $4.42 \pm 0.03$ & $4.34 \pm 0.03$ \\
\hline
\end{tabular}

a g/200g premezcla mineral: $\mathrm{KCl}, 28.57 ; \mathrm{MgSO}_{4} .7 \mathrm{H}_{2} \mathrm{O}, 28.57 ; \mathrm{ZnSO}_{4} .7 \mathrm{H}_{2} \mathrm{O}, 5.14 ; \mathrm{MnCl}_{2} .4 \mathrm{H}_{2} \mathrm{O}, 1.34 ; \mathrm{CuSO}_{4} .5 \mathrm{H}_{2} \mathrm{O}, 0.29 ; \mathrm{Kl}_{0} 0.29 ; \mathrm{CoCl}_{2} .2 \mathrm{H}_{2} \mathrm{O}, 0.14$; $\mathrm{Na}_{2} \mathrm{HPO}_{4}, 135.43$

${ }^{\mathrm{b}} \mathrm{g} / 900 \mathrm{~g}$ premezcla de vitaminas: Vitamina A acetato, $100000 \mathrm{UI}$; Vitamina $\mathrm{D}_{3}, 850 \mathrm{UI}$; Acetato di-alfa-tocoferol 2000 UI; menadiona, 2; tiamina-HCl, 0.5; rivoflavina $\left(\mathrm{B}_{2}\right)$, 3; piridoxina $\mathrm{HCl}\left(\mathrm{B}_{6}\right), 1$; DL-Ca-pantotenato, 5; ácido nicotínico, 5; biotina, 0.05; inositol, 5 ; Vitamina $\mathrm{B}_{12}$, 0.002 ; ácido fólico, 0.18 .

${ }^{\mathrm{c}}$ Extracto libre de nitrógeno $=100-(\%$ proteína cruda $+\%$ lípidos totales $+\%$ fibra cruda $+\%$ cenizas $)$.

La mezcla se pasó por un extrusor de $5 \mathrm{~mm}$ de diámetro. Los pellets se secaron por 8 horas a $65^{\circ} \mathrm{C}$ en un horno de convección. Estas dietas se conservaron en bolsas de plástico (Ziplock ${ }^{\circledR}$ ) a $-4^{\circ} \mathrm{C}$ hasta su uso.

Organismos y unidades experimentales. Se utilizaron 225 juveniles de $M$. tenellum $(0.31 \pm 0.01 \mathrm{~g}$ de peso y $32.62 \pm 1.10 \mathrm{~mm}$ de longitud total) obtenidos del medio natural y distribuidos aleatoriamente en 15 tinas de experimentales de plástico de 64 L (49x46×35 $\mathrm{cm} ; 15$ org/tina). Los organismos fueron seleccionados por su peso para homogenizar la población y se sometieron a una aclimatación durante 7 días, proporcionándoles a todos los organismos alimento con $30 \%$ PC. No se determinó el sexo de los organismos, ni se colocaron refugios en las tinas experimentales. Todas las tinas se mantuvieron con aguas claras y bajo condiciones controladas de oxígeno $(5.95 \pm 0.41 \mathrm{ppm})$, temperatura $\left(29.89 \pm 0.72^{\circ} \mathrm{C}\right)$, $\mathrm{pH}(8.44 \pm 0.15)$ y fotoperiodo (13:11 luz: oscuridad). Se emplearon filtros de cascada (Elite Hush 35) con capacidad de filtración de $500 \mathrm{~L} / \mathrm{h}$. El diseño experimental se basó en un modelo completamente al azar con cinco tratamientos con tres replicas para cada uno. Para evitar el efecto por el cambio de alimento, se acondicionó a los organismos durante una semana con la substitución parcial del alimento de inicio por cada uno de los alimentos experimentales, hasta llegar al $100 \%$ al fin de la semana. Durante los
60 días que duró el experimento los langostinos fueron alimentados una vez al día (14:00 h) con el $10 \%$ de su peso vivo para asegurar la saciedad. Se determina este porcentaje a priori por experiencias personales de los autores en estudios previos, donde no se ha observado un consumo de alimento superior al $6 \%$ en esta etapa de desarrollo. Las tinas se sifonearon seis horas después de la alimentación, para eliminar residuos de alimento, heces y mudas.

Parámetros biológicos evaluados. Los parámetros de crecimiento se calcularon a través de dos biometrías, para lo cual se midieron (Vernier; mm) y pesaron (balanza digital Scout pro OHAUS ${ }^{\circledR}$; g) todos los organismos al inicio y final del bioensayo. Para la determinación de la sobrevivencia se registraron diariamente los organismos muertos. El consumo total del alimento (CT) fue calculado del día 15 al 19 y 45 al 49 del bioensayo. Los parámetros biológicos fueron calculados de acuerdo a lo siguiente:

sobrevivencia $(\%)=100$ - (org. inicio - org. final / org. inicio) * 100;

cambio de talla $(\mathrm{mm} /$ día $)=$ talla final - talla inicial / días del bioensayos;

cambio de peso $(\mathrm{g} /$ día $)=$ peso final - peso inicial / días del bioensayos;

$\%$ incremento en longitud (IT \%) $=[$ (talla final 
- talla inicial) / talla inicial] * 100;

$\%$ incremento en peso (IP $\%)=[$ (peso final peso inicial) / peso inicial] * 100;

tasa de crecimiento específico de peso $(\mathrm{TCE})=[(\mathrm{In}$ peso final - In peso inicial) / días bioensayo]*100;

consumo total de alimento $(\mathrm{CT})=$ (alimento suministrado - alimento no consumido) / langostinos en la tina;

Consumo de $\mathrm{PC}$ total $(\mathrm{g} / \mathrm{org})=(\mathrm{PC}$ suministrada - PC consumida) / langostinos en la tina;

factor de conversión alimenticia $(F C A)=$ alimento consumido / incremento en peso;

tasa de eficiencia de la proteína $($ TEP $)=$ incremento en peso / proteína consumida.

Análisis estadístico. Los datos generados de sobrevivencia, peso y talla final, IP, IT, TCE, CT, FCA y TEP se les aplicó el análisis de varianza (ANOVA) de una vía para cada caso, previas pruebas de normalidad (KolmogorovSmirnov, $a=0.05$ ) y homocedasticidad (Bartlett, $a=0.05)$. Los datos expresados en porcentaje (sobrevivencia, IP y IT) se les aplicó la transformación del arcoseno de su raíz cuadrada (8). Las diferencias significativas entre las medias de los tratamientos se determinaron por medio del método de comparaciones múltiples de Tukey $(p<0.05)$. Todas las pruebas se realizaron mediante el software estadístico SigmaStat V3.1 (9).

\section{RESULTADOS}

Los resultados de los parámetros biológicos se muestran en la tabla 2. La sobrevivencia no tuvo diferencias estadísticamente significativas entre los tratamientos $(p>0.05)$, su promedio fue de $98.22 \pm 3.96 \%$. Los parámetros de crecimiento: peso final, talla final, cambio de peso y talla, IP, IT y TCE presentaron diferencias estadísticas $(p<0.05)$ entre los tratamientos. Los valores de peso y talla final fueron mayores para los organismos alimentados con $40 \%$ de PC en la dieta $(0.85 \pm 0.01 \mathrm{~g}$ y $41.61 \pm 0.47 \mathrm{~mm})$, presentando un cambio de peso de $0.54 \pm 0.02$ g y de talla de $9.19 \pm 1.37 \mathrm{~mm}$ en los 60 días del experimento. Los mayores valores para el IP, IT y TCE fueron en los langostinos alimentados con $40 \%$ de $P$ C, con $173.60 \pm 12.99 \%, 28.53 \pm 5.62 \%$ y $1.67 \pm 0.08$ respectivamente. El crecimiento más bajo se dio en los organismos alimentados con la dieta que contenía $20 \%$ de PC.

No existieron diferencias significativas $(p>0.05)$ en el CT y en la TEP entre los tratamientos. Fueron estadísticamente diferentes los tratamientos en sus valores de consumo de PC total y en el FCA $(p<0.05)$. Aunque no existió una diferencia en el CT y en la TEP, el consumo de PC total fue mayor en el tratamiento de $40 \%$ de PC $(0.52 \pm 0.01 \mathrm{~g} / \mathrm{org})$ respecto a lo consumido en el tratamiento de $20 \%$ de PC $(0.26 \pm 0.01 \mathrm{~g} / \mathrm{org})$. Para el FCA el mejor resultado lo obtuvieron los langostinos de los tratamientos del alimento de $35 \%$ de PC $(2.85 \pm 0.17)$ y de $40 \%$ de PC $(2.39 \pm 0.05)$. Existió una correlación favorable y positiva entre los resultados experimentales y la concentración porcentual de la PC de los alimentos experimentales (Tabla 3 ).

Tabla 2. Sobrevivencia, tasa de crecimiento específica, consumo de alimento, factor de conversión alimenticia y tasa de eficiencia proteica de juveniles de M. tenellum después de 60 días de tratamiento.

\begin{tabular}{|c|c|c|c|c|c|}
\hline \multirow{2}{*}{ Parámetros } & \multicolumn{5}{|c|}{ Tratamientos } \\
\hline & $20 \%$ PC & $25 \%$ PC & $30 \%$ PC & $35 \% \mathrm{PC}$ & $40 \% \mathrm{PC}$ \\
\hline Sobrevivencia (\%) & $100.0 \pm 0.00^{a}$ & $100.0 \pm 0.00^{\mathrm{a}}$ & $96.00 \pm 0.03^{a}$ & $99.00 \pm 0.02^{\mathrm{a}}$ & $100.00 \pm 0.00^{a}$ \\
\hline Peso inicial (g) & $0.31 \pm 0.00^{\mathrm{a}}$ & $0.31 \pm 0.01^{\mathrm{a}}$ & $0.31 \pm 0.00^{\mathrm{a}}$ & $0.32 \pm 0.01^{\mathrm{a}}$ & $0.31 \pm 0.01^{\mathrm{a}}$ \\
\hline Peso final $(\mathrm{g})$ & $0.56 \pm 0.02^{\mathrm{a}}$ & $0.62 \pm 0.02^{\mathrm{b}}$ & $0.70 \pm 0.01^{c}$ & $0.75 \pm 0.02^{\mathrm{c}}$ & $0.85 \pm 0.01^{\mathrm{d}}$ \\
\hline Cambio de peso (g/org) & $0.26 \pm 0.03^{a}$ & $0.31 \pm 0.01^{\mathrm{b}}$ & $0.39 \pm 0.02^{c}$ & $0.43 \pm 0.02^{c}$ & $0.54 \pm 0.02^{\mathrm{d}}$ \\
\hline Talla inicial (mm) & $32.18 \pm 0.74^{\mathrm{a}}$ & $32.29 \pm 0.77^{a}$ & $32.78 \pm 1.16^{\mathrm{a}}$ & $32.76 \pm 0.60^{\mathrm{a}}$ & $32.42 \pm 1.70^{\mathrm{a}}$ \\
\hline Talla final (mm) & $36.96 \pm 0.97^{a}$ & $37.86 \pm 0.58^{\mathrm{ab}}$ & $38.16 \pm 0.63^{\mathrm{ab}}$ & $39.36 \pm 0.30^{\mathrm{b}}$ & $41.61 \pm 0.47^{c}$ \\
\hline Cambio de talla (mm/org) & $4.79 \pm 1.64^{\mathrm{a}}$ & $5.57 \pm 0.22^{\mathrm{a}}$ & $5.39 \pm 1.29^{a}$ & $6.60 \pm 0.43^{\mathrm{ab}}$ & $9.19 \pm 1.37^{b}$ \\
\hline IP (\%) & $82.14 \pm 8.74^{a}$ & $99.16 \pm 3.00^{a}$ & $127.32 \pm 4.76^{b}$ & $134.00 \pm 7.69^{b}$ & $173.6 \pm 12.99^{c}$ \\
\hline IT $(\%)$ & $14.96 \pm 5.44^{a}$ & $17.28 \pm 1.07^{\mathrm{ab}}$ & $16.53 \pm 4.37^{\mathrm{ab}}$ & $20.18 \pm 1.66^{\mathrm{ab}}$ & $28.53 \pm 5.62^{b}$ \\
\hline TCE & $0.99 \pm 0.08^{a}$ & $1.15 \pm 0.025^{\mathrm{a}}$ & $1.37 \pm 0.03^{b}$ & $1.41 \pm 0.05^{\mathrm{b}}$ & $1.67 \pm 0.08^{c}$ \\
\hline CT (g/org) & $1.24 \pm 0.06^{\mathrm{a}}$ & $1.31 \pm 0.27^{\mathrm{a}}$ & $1.41 \pm 0.15^{\mathrm{a}}$ & $1.21 \pm 0.13^{\mathrm{a}}$ & $1.30 \pm 0.03^{a}$ \\
\hline Consumo PC total (g/org) & $0.26 \pm 0.01^{\mathrm{a}}$ & $0.33 \pm 0.07^{\mathrm{ab}}$ & $0.42 \pm 0.04^{\mathrm{bc}}$ & $0.42 \pm 0.04^{\mathrm{bc}}$ & $0.52 \pm 0.01^{c}$ \\
\hline FCA & $5.18 \pm 0.71^{a}$ & $4.23 \pm 0.81^{\mathrm{ab}}$ & $3.57 \pm 0.25^{\mathrm{bc}}$ & $2.82 \pm 0.17^{c}$ & $2.39 \pm 0.05^{c}$ \\
\hline TEP & $0.97 \pm 0.13^{a}$ & $0.97 \pm 0.18^{\mathrm{a}}$ & $0.93 \pm 0.07^{a}$ & $1.01 \pm 0.06^{\mathrm{a}}$ & $1.04 \pm 0.02^{\mathrm{a}}$ \\
\hline
\end{tabular}

Los valores medios \pm DE seguidos por superíndices diferentes en las fila indican diferencias significativas $(p<0.05)$. 
Tabla 3. Correlación de resultados experimentales y el porcentaje de proteína cruda en los alimentos experimentales.

\begin{tabular}{|c|c|c|}
\hline $\begin{array}{c}\text { Parámetros } \\
\text { biológicos }\end{array}$ & $\begin{array}{l}\text { Ecuación de la línea } \\
\text { de regresión linear }\end{array}$ & $\begin{array}{c}\text { Correlación } \\
\left(\mathbf{R}^{2}\right)\end{array}$ \\
\hline Peso final ( $g$ ) & $y=0.014 x+0.2782$ & 0.9622 \\
\hline $\begin{array}{l}\text { Cambio de peso } \\
\text { (g/org) }\end{array}$ & $y=0.0002 x-0.0005$ & 0.9518 \\
\hline Talla final (mm) & $y=0.2156 x+32.325$ & 0.8199 \\
\hline $\begin{array}{l}\text { Cambio de talla } \\
\text { (mm/org) }\end{array}$ & $y=0.0033 x+0.0069$ & 0.5894 \\
\hline $\begin{array}{l}\text { Incremento en peso } \\
(\mathrm{IP} ; \%)\end{array}$ & $y=4.3553 x-7.4153$ & 0.9187 \\
\hline $\begin{array}{l}\text { Incremento en talla } \\
\text { (IT; \%) }\end{array}$ & $y=0.6005 x+1.4781$ & 0.5229 \\
\hline $\begin{array}{l}\text { Tasa de Crecimiento } \\
\text { Especifica (TCE) }\end{array}$ & $y=0.0325 x+0.3467$ & 0.9305 \\
\hline $\begin{array}{l}\text { Consumo de PC total } \\
\text { (g/org) }\end{array}$ & $y=0.0002 x+0.0004$ & 0.8293 \\
\hline $\begin{array}{l}\text { Factor de Conversión } \\
\text { alimenticia (FCA) }\end{array}$ & $y=-0.1396 x+7.8286$ & 0.8408 \\
\hline
\end{tabular}

\section{DISCUSIón}

La sobrevivencia de los juveniles de $M$. tenellum, del presente estudio, fue de $98.22 \pm 3.96 \%$ independientemente del nivel de proteína con que fueron alimentados. Resultados similares reportaron García-Ulloa Gómez et al (7), al alimentar por 45 días con base en una dieta de $40 \%$ de PC a juveniles de esta especie $(91.66 \%$ de sobrevivencia); y por Gitte y Indulkar (10) quienes registraron sobrevivencias del $82.20 \pm 3.56 \%$ en $M$. rosenbergii alimentados por 30 días con una dieta que contenía el $40 \%$ de PC. Mientras que Luna et al (11), reportan las mejores sobrevivencias (70\%) de su estudio en organismos alimentados por 30 días con $25 \%$ de PC; contradictoriamente, Abbaspour-Davassi (12) al evaluar cinco dietas con diferentes contenidos en proteína $(15,30,45,60,75 \%$ de PC) en juveniles de $M$. rosenbergii, obtiene la más baja sobrevivencia en su tratamiento con $75 \%$ de PC (13.33\%). En sistemas de cultivo experimental de langostinos alimentados con $35-40 \%$ de $P C$, tanto $M$. tenellum como $M$. rosenbergii, se han reportado sobrevivencias de 70 a $90 \%(6,13,14)$, influenciadas en ocasiones por la inclusión de refugios en el sistema $(7,13)$. Cuando las sobrevivencias son bajas pueden deberse al canibalismo por falta de refugios para los organismos que se encuentran en fase de muda (11), cuestión que no se reflejó en el presente estudio.

En esta investigación los valores de los parámetros de crecimiento (cambio de peso y talla, IP, IT y TCE) mostraron una tendencia positiva, en la cual el crecimiento de los organismos estuvo relacionado directamente con el porcentaje de proteína cruda suministrado; los organismos que crecieron más fueron los del tratamiento con alimento con $40 \%$ de PC. El cambio de peso después de los 60 días del bioensayo fue de $0.54 \pm 0.02 \mathrm{~g}$ para el tratamiento de $40 \%$ de PC, coincide con lo obtenido por García-Ulloa Gómez et al (7) a los 45 días con el mismo langostino y nivel de PC en la dieta $(0.53 \pm 0.24$ g). En M. rosenbergii existen resultados similares al presente trabajo, en los que se reportan los mejores parámetros de crecimiento (cambio de peso y talla, IP, IT y TCE) con dietas de 35 a $45 \%$ de PC $(12,13,14)$. Casos contrarios han sido señalados, donde los mejores parámetros de crecimiento para juveniles de este género se han encontrado al alimentar con niveles proteicos iguales o inferiores al $25 \%$ de PC, como lo mencionado por Luna et al (11) en postlarvas de $M$. rosenbergii, al evaluar tres dietas con 25 , 28 y $35 \%$ de PC, quienes obtuvieron un mayor crecimiento en longitud y cambio de peso con la dieta de $25 \%$ de PC; mientras que Urbano et al (15) reportan, en cultivos experimentales de $M$. jelskii mayores crecimientos en longitud y peso a los 100 días con dietas con $20 \%$ de PC; y De los Santos y Silva (16), en su estudio de M. michoacanus bajo dietas con 25 y $28 \%$ de $P C$, que no encontraron diferencias significativas $(p<0.05)$ en el cambio de peso durante 5 meses entre las dos dietas (1.17 y $1.22 \mathrm{~g}$, respectivamente).

La tendencia de incremento de los valores del crecimiento de los juveniles de $M$. tenellum conforme al incremento de PC en las dietas de esta investigación, pueden deberse al óptimo aprovechamiento de la proteína de la dieta que se acerca a cubrir el requerimiento proteico en esta etapa de crecimiento exponencial (cercano al $40 \%$ de $\mathrm{PC}$ ), el cuál posiblemente disminuirá al acercarse a la etapa adulta, en la que se menciona para esta especie como requerimiento proteico no más del $29 \%$ de PC $(5,17)$. De los Santos y Silva (16), mencionan que el crecimiento entre langostinos no es uniforme y que dependerá de la especie y del tiempo en el cual se completa su ciclo de vida, esa es la velocidad con la que se va a crecer.

Esta tendencia de crecimiento con respecto a la proteína suministrada posiblemente se pueda explicar por el incrementó en el consumo de PC y la mejora del FC cuando se aumenta el porcentaje de PC en la dieta, aunque el CT y el TEP no muestren diferencias. Indicando con ello, que los organismos que reciben un nivel más alto de PC aprovechan mejor las dietas suministradas. El mejor resultado en el FCA se obtuvo en los langostinos alimentados con las dietas de 35 y $40 \%$ de PC $(2.85 \pm 0.17$ y $2.39 \pm 0.05$, respectivamente), similar a lo reportado en $M$. rosenbergii por Kabir et al (13) en alimentos de 
35 y $40 \%$ de PC y por Hasanuzzaman et al (18) en alimentos con $25 \%$ de PC ( 2.21 y 2.63 ; y 2.27 , respectivamente). Estudios en $M$. tenellum reportaron valores del FCA de 1.5 en dietas con $25 \%$ de PC (19) y 1.40 en dietas con $40 \%$ de PC (7). Según Kabir et al (13), mencionan que altos valores de FCA (en el orden de 2.5-2.6) podría indicar que la proteína se estaría utilizando como energía, contraponiéndose a los resultados del presente estudio, en los cuales los menores crecimientos corresponden a los FCA más altos. En M. tenellum aún no se establecen los requerimientos óptimos de proteína; sin embargo, se menciona que los juveniles de esta especie han tenido crecimientos favorables con $40 \%$ de proteína en sus dietas (5). Ya que se destacó a la proteína como el principal componente de las dietas comerciales, debido a su gran influencia sobre el crecimiento (20), algunos investigadores recomiendan usar raciones con un contenido proteico mayor al $40 \%$ para la alimentación de camarones de agua dulce (21). Lo anteriormente reportado y los resultados obtenidos en la presente investigación demuestran que en juveniles de $M$. tenellum conforme se aumenta el nivel de la proteína en la dieta se mejora el crecimiento de los organismos, y que para esta etapa $40 \%$ de PC en las dietas es el conveniente. Por lo tanto, se puede concluir que, bajo las condiciones experimentales aquí establecidas, juveniles de $M$. tenellum alimentados con $40 \%$ de PC se desarrollan más rápido que organismos que reciban una menor concentración de este nutriente. Se recomienda realizar investigaciones más específicas que permitan acotar el rango de requerimiento proteico en esta etapa de crecimiento, así como desarrollar alimentos específicos para la especie.

\section{Agradecimientos}

Se agradece al Consejo Nacional de Ciencia y Tecnología (CONACYT-Beca 34091) y al Consejo Estatal de Ciencia y Tecnología de Jalisco (COECYTJAL - Proyecto 06-2009-661) por apoyar esta investigación. A los integrantes del Laboratorio de Acuicultura Experimental del Centro Universitario de la Costa-UdeG por su apoyo.

\section{REFERENCIAS}

1. Ponce-Palafox JT, Arana-Magallón FC, Cabanillas BH, Esparza LH. Bases biológicas y técnicas para el cultivo de los camarones de agua dulce nativos del Pacífico Americano Macrobrachium tenellum (Smith, 1871) y M. americanum (Bate, 1968). CIVA (en línea) 2002 (fecha de acceso 30 de octubre de 2011); 1: 534-536. URL disponible en: http://www.revistaaquatic.com/civa2002/ coms/pdf/67.pdf

2. Espinosa-Chaurand LD, Vargas-Ceballos M, Guzmán-Arroyo M, Nolasco-Soria H, Carrillo-Farnés O, Chong-Carrillo $\mathrm{O}$ et al. Biología y cultivo de Macrobrachium tenellum: Estado del arte. Hidrobiológica 2011; 21(2): 99-117.

3. Vega-Villasante $F$, Espinosa CLD, Yamasaki GS, Cortés JE, García GMU, Cupul MAL, et al. Acuicultura del langostino Macrobrachium tenellum - Engorda de estanques semirrústicos. Jalisco, México: Universidad de Guadalajara; 2011.
4. Vega-Villasante F, Chong CO. El Dicamarón, Diccionario de camaronicultura. 2 ed. Jalisco, México: Universidad de Guadalajara; 2006.

5. Jayachandran KV. Palaemonid Prawns: Biodiversity, taxonomy, biology and management. Enfield $\mathrm{NH}$, USA: Science Publishers Inc; 2001.

6. García Ulloa-Gómez M, López-Aceves LA, Ponce-Palafox JT, Rodríguez-González $\mathrm{H}$, Arredondo-Figueroa JL. Growth of freshwater prawn Macrobrachium tenellum (Smith, 1871) juveniles fed isoproteic diets substituting fish meal by soya bean meal. Braz Arch Biol Technol 2008; 5(1): 57-65.

7. Mambrini M, Guillaume J. Protein nutrition. En: Guillaume J, Kaushik S, Bergot P, Métailler $\mathrm{R}$ (eds): Nutrition and feeding on fish and crustaceous. Chichester UK: Springer and praxis publishing; 2011.

8. Zar JH. Bioestadistical Analysis. 4 edition. New Jersey, USA: Prentice-Hall; 1999. 
9. SigmaStat, Advisory Statistics for Scientists (programa de computadora). Version 3.1. Chicago (IL): SYSTAT software inc; 2004.

10. Gitte MJ, Indulkar ST. Evaluation of marine fish meat incorporated diets on growth and survival of post-larvae of Macrobrachium rosenbergii (de Man). Asian Fish Sci 2005; 18(2005): 323-334.

11. Luna M, Graziani C, Villarroel E, Lemus M, Lodeiros C, Salazar G. Evaluación de tres dietas con diferente contenido proteico en el cultivo de postlarvas del langostino de río Macrobrachium rosenbergii. Zootecnia Trop 2007; 25(2): 111-121.

12. Abbaspour-Davassi L. Survival and growth of the freshwater prawn Macrobrachium rosenbergii in relation to different nutrients composition. J Fish Aquat Sci 2011; 6(6): 649-654.

13. Kabir Chowdhury MA, Goda AMAS, EIHaroun ER, Wafa MA, Salah El-Din SA. Effect of dietary protein and feeding time on growth performance and feed utilization of post larval freshwater prawn Macrobrachium rosenbergii (de Man 1879). J Fish Aquat Sci 2008; 3(1): 1-11.

14. Vega-Villasante F, Martínez-López EA, Espinosa-Chaurand LD, Cortés-Lara MC, Nolasco-Soria H. Crecimiento y supervivencia del langostino (Macrobrachium tenellum) en cultivos experimentales de verano y otoño en la costa tropical del Pacífico mexicano. Trop Subtrop Agroecosyst 2011; 14: 581-588.

15. Urbano T, Silva-A A, Medina L, Moreno C, Guevara M, Graziani C. Crecimiento del camarón de agua dulce Macrobrachium jelskii (Miers, 1877), en lagunas de cultivo. Zootecnia Trop 2010; 28(2): 163-171.
16. De los Santos Romero R, Silva Rivera ME. Crecimiento de Macrobrachium michoacanus con relación al tipo de alimento y densidad de cultivo. Naturaleza y Desarrollo 2008; 6(1): $16-25$.

17. Guzmán Arroyo M. Biología, ecología y pesca del langostino Macrobrachium tenellum (Smith, 1871), en lagunas costeras del estado de Guerrero, México (tesis doctoral). D.F., México: Universidad Autónoma de México; 1987.

18. Hasanuzzaman AF, Siddiqui N, Chisty $\mathrm{AH}$. Optimum remplacement of fishmeal with soybean meal in diet for Macrobrachium rosenbergii (de Man 1879) culture in low saline water. Turk J Fish Aquat Sci 2009; 9: 17-22.

19. Ponce-Palafox JT, García-Ulloa GM, Arredondo FJL, Hernández OD, Díaz AJ, Aldama RG, et al. El cultivo del camarón de agua dulce Macrobrachium tenellum en estanques rústicos. CIVA (en línea) 2006 (fecha de acceso 30 de octubre de 2011); 4: 655-660. URL disponible en: http:// www. revistaaquatic.com/civa2006/coms/ pdf/246.pdf

20. Teshima S-I, Koshio S, Ishikawa M, Shah M, Hernandez Hernandez LH. Protein requirements of the freshwater prawn Macrobrachium rosenbergii evaluated by the factorial method. J World Aquac Soc 2006; 37: 145-153.

21. Valenti WC. Comportamento reprodutivo de camarões de água doce. Encontro Anual de Etologia; 1987 oct 1-3; Jaboticabal, Sao Pablo, Brasil. Jaboticabal, Sao Pablo, Brasil: FUNEP; 1987. 